\title{
Conocimiento, actitudes y experiencia respecto a trauma dental en estudiantes de odontología en Cartagena, Colombia
}

\author{
Knowledge, Attitudes and Experience Regarding Dental \\ Trauma in Dentistry Students in Cartagena, Colombia
}

Irene Margarita Lora Salgado, Esp. ${ }^{1}$, Lesbia Rosa Tirado Amador $\bowtie$, Esp. ${ }^{2}$, Édgar Daniel Vargas Quiroga, Od. ${ }^{3}$, Demetrio Barcha Barreto, MSc. ${ }^{4}$, Leidy Lombana Ortiz, Od. ${ }^{5}$, Mariangélica Ramírez Pattigno, Od. ${ }^{6}$, Aníbal Estrada Martínez, Od. ${ }^{7}$, Germán Ortiz Brun, Od. ${ }^{8}$

\author{
${ }^{1}$ Universidad del Sinú, Cartagena, Colombia \\ 2 Universidad de Cartagena, Cartagena, Colombia \\ ${ }^{3}$ Universidad del Sinú, Cartagena, Colombia \\ ${ }^{4}$ Universidad del Sinú, Cartagena, Colombia \\ ${ }^{5}$ Universidad del Sinú, Cartagena, Colombia \\ ${ }^{6}$ Universidad del Sinú, Cartagena, Colombia \\ 7 Universidad del Sinú, Cartagena, Colombia \\ ${ }^{8}$ Universidad del Sinú, Cartagena, Colombia
}

Escuela de Odontología, Universidad del Sinú, Avenida Pedro de Heredia n. ${ }^{\circ} 31-176$ sector Amberes, Cartagena, Colombia. Correo electrónico: investigacionesodontologia@unisinucartagena.edu.co.

Recibido: 3 de mayo del 2017 Aprobado: 15 de junio del 2017

Cómo citar este artículo: Lora-Salgado IM, Tirado-Amador LR, Vargas-Quiroga ED, Barcha-Barreto D, Lombana-Ortiz L, Ramírez-Pattigno M, et al. Conocimiento, actitudes y experiencia respecto a trauma dental en estudiantes de odontología en Cartagena, Colombia. Rev Nac Odontol. 2018;13(26):1-11. doi: http://dx.doi.org/10.16925/od.v13i26.2043

\section{Resumen}

Introducción: los traumatismos dentales se presentan como motivo de consulta odontológica, con frecuencia en niños y jóvenes. Esto provoca impacto social y psicológico. Diversos estudios se interesan en el conocimiento de padres, maestros y odontólogos sobre el tema. Para el caso de estudiantes de odontología, no se dispone de mucha información. Por ello, quiso determinarse el nivel de conocimiento, actitudes y experiencia en la atención del trauma dental en estudiantes de una escuela universitaria privada en Cartagena, Colombia.

Métodos: estudio descriptivo de corte transversal en estudiantes de último año, por medio de un cuestionario de autorreporte anónimo. Los datos fueron procesados y analizados con el programa Stata v.13.2, utilizando estadística descriptiva y análisis bivariado a través de pruebas $\chi^{2}$.

Resultados: la muestra fue de 45 sujetos, con edad promedio 23,3 $\pm 1,94$ años. El nivel de conocimiento fue aceptable en $64,4 \%$ de los estudiantes, y asimismo los estudiantes que forman parte de este porcentaje presentan un escaso conocimiento en fractura alveolar, luxación intrusiva con fístula supurante, luxación intrusiva y lateral y según la severidad del trauma. Las actitudes fueron desfavorables en $82 \%$ de los estudiantes y el $95 \%$ no ha cursado con casos de traumatismos dentales. No se reportó asociación estadísticamente significativa entre variables de estudio.

Conclusión: aunque el nivel de conocimiento respecto a trauma dental en los sujetos de estudio es aceptable y es notoria la poca experiencia clínica en su tratamiento, es compromiso de la Escuela de Odontología propender desde la academia a fortalecer las competencias en la atención de este tipo de situaciones clínicas.

Palabras clave: actitudes y prácticas en salud (DeCS-Bireme), conocimientos, estudiantes de odontología, traumatismos en los dientes. 


\title{
Knowledge, Attitudes and Experience Regarding Dental Trauma in Dentistry Students in Cartagena, Colombia
}

\begin{abstract}
Introduction: Dental trauma is a reason for dental consultation, often in children and young people. It has social and psychological impacts. Several studies are interested in the knowledge of parents, teachers and dentists on the subject. In the case of dentistry students, not much information is available. Therefore, we wanted to determine the level of knowledge, attitudes and experience regarding dental trauma care of students from a private university in Cartagena, Colombia.

Materials and methods: Cross-sectional, descriptive study in senior students through an anonymous self-report questionnaire. The data were processed and analyzed by Stata v. 13.2, using descriptive statistics and bivariate analysis through $\chi^{2}$ tests.

Results: The sample was 45 subjects, with an average age of $23.3 \pm 1.94$ years old. The level of knowledge was acceptable in $64.4 \%$ of the students, and the students who are part of this percentage have poor knowledge of alveolar fracture, intrusive dislocation with suppurating fistula, intrusive and lateral dislocation and according to the severity of trauma. Attitudes were unfavorable in $82 \%$ of the students and $95 \%$ had not seen dental trauma cases. No statistically significant association was reported among study variables.

Conclusion: While the level of knowledge regarding dental trauma in the study subjects is acceptable and the little clinical experience in its treatment is notorious, the School of Dentistry is required to promote from academia the strengthening of skills to deal with these clinical situations.
\end{abstract}

Keywords: attitudes and practices (DeCS-Bireme), health knowledge, dentistry students, dental trauma.

\section{Conhecimento, atitudes e experiência quanto ao trauma dental em estudantes de odontologia em Cartagena, Colômbia}

\section{Resumo}

Introdução: os traumatismos dentais são motivo de consulta odontológica com frequência entre crianças e jovens. Isso provoca impacto social e psicológico. Diversos estudos se interessam no conhecimento de pais, professores e odontologistas sobre o tema. Para o caso de estudantes de odontologia, não se dispõe de muita informação. Portanto, pretendeu-se determinar o nível de conhecimento, atitudes e experiência no atendimento do trauma dental em estudantes de uma escola universitária particular em Cartagena, Colômbia.

Métodos: estudo descritivo, de corte transversal, com estudantes do último ano, por meio de um questionário de autorrelato anônimo. Os dados foram processados e analisados com o programa Stata v.13.2 e utilizadas estatística descritiva e análise bivariada por meio de testes $\chi^{2}$.

Resultados: a amostra foi de 45 sujeitos, com idade média de 23,3 $\pm 1,94$ anos. 0 nível de conhecimento foi aceitável em $64,4 \%$ dos estudantes e, além disso, os que fazem parte dessa porcentagem apresentam um escasso conhecimento em fratura alveolar, luxação intrusiva com fístula supurante, luxação intrusiva e lateral e segundo a gravidade do trauma. As atitudes foram desfavoráveis em 82\% dos estudantes, e 95\% não cursaram com casos de traumatismos dentais. Não se relatou associação estatisticamente significativa entre variáveis de estudo.

Conclusão: embora o nível de conhecimento a respeito do trauma dental nos sujeitos de estudo seja aceitável e seja notável a pouca experiência clínica em seu tratamento, é compromisso da Escola de Odontologia propender a partir da academia para fortalecer as competências no atendimento desse tipo de situações clínicas.

Palavras-chave: atitudes e práticas em saúde (DeCS-Bireme), conhecimentos, estudantes de odontologia, traumatismos nos dentes. 


\section{Introducción}

La atención odontológica ha centrado su enfoque de acción en ciertas situaciones que constituyen problemas de salud pública, como la caries dental y la enfermedad periodontal. No obstante, otras situaciones como las lesiones traumáticas se han convertido en un problema de relevancia social, y se destaca el traumatismo dental como uno de los tipos de lesiones más frecuentes en niños y jóvenes, en un rango de 12,0-64,8\% [1], lo que produce gran impacto social y psicológico $[2,3]$.

A nivel físico, generan impacto negativo en el sistema estomatognático al influir en la función y estética que brindan los dientes, pues pueden limitarse solo al daño de la integridad de la estructura dental o comprometer hueso y otros tejidos de sostén; además de la posibilidad de inducir desplazamiento total o parcial de la posición dental habitual [4]. Con respecto a esto último, algunos factores de la oclusión como la presencia de overjet aumentado y mordida abierta han sido asociados como factores de riesgo para experiencia de trauma dental en denticiones permanente y primaria [5].

La epidemiología del traumatismo dental evidencia que suele afectar más a hombres que a mujeres, en situaciones cotidianas. Entre los factores etiológicos asociados con mayor frecuencia, es posible mencionar el impacto físico contra los órganos dentarios tras la exposición a caídas, la práctica de deportes, los enfrentamientos y accidentes entre personas o el impacto con algunos objetos [6]. En referencia a los traumatismos dentales mayormente reportados, se destacan las fracturas de esmalte y las fracturas de esmalte-dentina sin exposición pulpar [7-9].

Diversos estudios han centrado su interés en el conocimiento de padres, maestros y odontólogos sobre el tema de traumatismos dentales, y han destacado la importancia de educar al respecto para contribuir a mejores pronósticos de dientes expuestos a traumatismo [10-13]. Sin embargo, dado que la atención profesional de las condiciones dentales es competencia del odontólogo, se considera necesario disponer de ciertos conocimientos y actitudes básicas para brindar la atención pertinente en caso de traumatismo dental. Esto se debe a que dicha atención no se desarrolla solo a partir de la experiencia profesional, sino que además es producto de un proceso que involucra el recorrido de los odontólogos durante su etapa de formación académica, que consiste en un proceso pedagógico único que permite la adquisición de conocimiento teórico, habilidades clínicas e interpersonales [14].

De tal modo, será muy importante identificar las falencias de los estudiantes de odontología en conceptos y protocolos de las diferentes situaciones clínicas de traumatismo dental, más aún cuando se encuentran cursando el último año académico, próximos a graduarse y a convertirse en profesionales, pues la academia puede ser un gran escenario para corregir errores y otorgar argumentos sólidos que propicien el desarrollo de las habilidades y competencias para una atención adecuada.

Entre los estudiantes de Odontología de la Universidad del Sinú, seccional Cartagena, de último año académico, existe una particular condición y es haber cursado en semestres anteriores la totalidad de asignaturas teóricas en las que se imparte la temática de traumatismo dental, desde aspectos concernientes a su epidemiología, diagnóstico clínico y radiográfico, hasta su tratamiento; además de poseer una experiencia superior a un año en el escenario clínico. Esto reviste el interés de los investigadores para plantear la presente investigación, con el objetivo de determinar el nivel de conocimiento, actitudes y experiencia en la atención de trauma dental en la comunidad estudiantil de último año de la Escuela de Odontología de la Universidad del Sinú.

\section{Materiales y métodos}

\section{Selección y descripción de los participantes}

Se realizó un estudio descriptivo de corte transversal, en una población constituida por 54 estudiantes de último año (correspondiente a noveno y décimo semestre) de la Escuela de Odontología de la Universidad del Sinú, seccional Cartagena, durante el periodo comprendido entre febrero y mayo del 2015. Finalmente, la muestra total estuvo conformada por 45 estudiantes que de manera voluntaria se vincularon a la investigación, dado que tres estudiantes no desearon participar y siete fueron excluidos por tener la calidad de estudiantes irregulares (cursaban solo algunas de las asignaturas de los semestres incluidos en el presente estudio). Para el diseño del estudio y la recolección de información, se consideró la normatividad nacional vigente en Colombia para investigación 
en el área de la salud, consignada en la Resolución 008430 de 1993, la cual dispone que esta investigación corresponde a riesgo mínimo [15], y se obtuvo la autorización de los participantes mediante formato escrito de consentimiento informado.

\section{Información técnica}

\section{Instrumento para recolección de información}

Para la obtención de información sociodemográfica y medición de las variables de conocimientos, actitudes y experiencia respecto a trauma dental, se implementó un formato en el que se registró la edad y el sexo de cada participante, y que incluyó un cuestionario estructurado anónimo de autorreporte, utilizando argumentos teóricos consignados en la guía electrónica de trauma dental, denominada The Dental Trauma Guide (diseñada por el hospital Rigshospitalet de Dinamarca y la International Association of Dental Traumatology) [16]. Su diseño y calibración se realizó mediante juicio de expertos en la temática para verificación de la validez de apariencia y contenido, tras lo cual se logró suficiencia y pertinencia de las categorías, con un resultante valor estadístico Kappa Cohen de 0,70 , que implica una concordancia considerable.

Este cuestionario estuvo conformado por 17 preguntas (selección múltiple con única respuesta correcta), las cuales fueron distribuidas en un total de 10 preguntas sobre trauma dental: nueve orientadas a la evaluación del nivel de conocimiento y una para identificar la asignatura de la cual los estudiantes consideran que proviene su conocimiento sobre trauma dental.

Para la variable de nivel de conocimiento, se incluyó conocimiento referente a diagnóstico de fractura coronal, infracción, concusión, fractura alveolar, luxación intrusiva con fístula supurante, luxación extrusiva, luxación intrusiva, luxación lateral con movilidad leve y severidad de trauma dental. Las preguntas estaban formuladas a manera de casos clínicos, con múltiples opciones de respuesta y solo una opción correcta. Se establecieron tres indicadores (de una a tres preguntas correctas: escaso conocimiento; de cuatro a seis preguntas correctas: conocimiento aceptable; y de siete a nueve preguntas correctas: conocimiento bueno).
Para la variable de tipo de actitudes, se utilizaron seis preguntas formuladas como caso clínico y con la posibilidad de seleccionar una única solución a partir de opciones múltiples. Estas preguntas estuvieron enfocadas en el protocolo de atención en subluxación, actitud en caso de fractura radicular, fisura en tercio medio, protocolo de tratamiento de urgencia en avulsión, fractura coronal no complicada, fractura coronal no complicada con ápice abierto, y actitud a seguir en caso de infracción; y hubo una pregunta para identificar la experiencia clínica en atención de trauma dental. Se consideró un nivel favorable al responder correctamente de cuatro a siete preguntas de actitudes.

Para la recolección de información, dos individuos fueron estandarizados con los expertos que diseñaron el instrumento en la comprensión de su contenido para aclarar cualquier duda a los sujetos participantes.

Para lograr la estandarización de los procesos de recolección y determinar la comprensión de las preguntas, se realizó una prueba piloto en cinco estudiantes seleccionados aleatoriamente y que después fueron excluidos de la muestra final del estudio. Luego se hicieron las modificaciones necesarias a las preguntas y se procedió a la aplicación del instrumento.

\section{Estadística}

La información recolectada se tabuló en una matriz de datos en el software Excel versión 2010, y fue transportada al software Stata v.13.2 para Windows 11.0 (Stata Corp., TX, EE. UU.). Los datos fueron analizados a través de estadística descriptiva (frecuencias y porcentajes) y análisis bivariado, a fin de establecer asociación entre las variables sociodemográficas y nivel de conocimientos y actitudes, empleando pruebas $\chi^{2}$ con un límite de significancia de 0,05 .

\section{Resultados}

Un total de 45 estudiantes conformaron la población de estudio, en el que poco más de la mitad de los participantes fueron mujeres y la edad promedio de los sujetos fue 23,3 $\pm 1,94$ años. En la Tabla 1, se presentan las características sociodemográficas. 
Tabla 1. Caracterización sociodemográfica de los sujetos de estudio

\begin{tabular}{lcc}
\hline $\begin{array}{c}\text { Características } \\
\text { demográficas }\end{array}$ & Frecuencia & Porcentaje\% \\
\hline Edad & 28 & 62,22 \\
De 21 a 23 años & 14 & 31,11 \\
De 24 a 26 años & 3 & 6,66 \\
$>$ 27 años & $\mathbf{4 5}$ & $\mathbf{1 0 0}$ \\
\hline Total & & 64,44 \\
\hline Sexo & 29 & 35,55 \\
Femenino & 16 & $\mathbf{1 0 0}$ \\
Masculino & $\mathbf{4 5}$ & \\
\hline Total & & \\
\hline
\end{tabular}

Fuente: elaboración propia
En referencia a conocimientos específicos para los diferentes tipos de trauma dental, más de la mitad de los sujetos no posee conocimiento sobre los diferentes tipos de luxación, fractura alveolar ni severidad de trauma dental. Poco más del $50 \%$ de los sujetos conoce sobre infracción del esmalte (53\% IC 95\% =33-68) y concusión ( $66 \%$ IC $95 \%=52-80)$. Con respecto al conocimiento adquirido de trauma dental, un $63 \%$ refiere que proviene del área de endodoncia (IC 95=33-68; Tabla 2).

Las actitudes fueron desfavorables en más del $60 \%$ de los sujetos en cuanto al protocolo de acción relacionado con fractura radicular, avulsión, fractura de corona no complicada sin ápice abierto e infracción. En cuanto a la experiencia clínica, el 95\% de los estudiantes no ha asumido acciones en traumas dentales (Tabla 3).

Tabla 2. Descriptivos de conocimiento en referencia a trauma dental en estudiantes de la Escuela de Odontología, Universidad del Sinú, Cartagena, 2015

\begin{tabular}{|c|c|c|c|}
\hline Preguntas relacionadas con el conocimiento & Frecuencia & $\%$ & $I C=95 \%$ \\
\hline \multicolumn{4}{|l|}{ P2: Conocimiento de fractura coronal } \\
\hline Conoce & 32 & 71 & $57-84$ \\
\hline No conoce & 13 & 28 & $15-42$ \\
\hline \multicolumn{4}{|l|}{ P5: Conocimiento de infracción } \\
\hline Conoce & 24 & 53 & $38-68$ \\
\hline No conoce & 21 & 46 & $31-61$ \\
\hline \multicolumn{4}{|l|}{ P6: Conocimiento de concusión } \\
\hline Conoce & 30 & 66 & $52-80$ \\
\hline No conoce & 15 & 33 & $19-47$ \\
\hline \multicolumn{4}{|l|}{ P7: Conocimiento de fractura alveolar } \\
\hline Conoce & 13 & 28 & $15-42$ \\
\hline No conoce & 32 & 71 & $57-84$ \\
\hline \multicolumn{4}{|l|}{ P10: Conocimiento de luxación intrusiva. Fístula supurante } \\
\hline Conoce & 18 & 40 & $25-54$ \\
\hline No conoce & 27 & 60 & 45-74 \\
\hline \multicolumn{4}{|l|}{ P11: Conocimiento de luxación extrusiva } \\
\hline Conoce & 22 & 48 & $33-64$ \\
\hline No conoce & 23 & 51 & $35-66$ \\
\hline \multicolumn{4}{|l|}{ P12: Conocimiento de luxación intrusiva } \\
\hline Conoce & 13 & 28 & $15-42$ \\
\hline No conoce & 32 & 71 & $57-84$ \\
\hline \multicolumn{4}{|l|}{ P13: Conocimiento de luxación lateral. Movilidad leve } \\
\hline Conoce & 9 & 20 & $7-32$ \\
\hline No conoce & 36 & 80 & $67-92$ \\
\hline
\end{tabular}


(viene)

\begin{tabular}{|c|c|c|c|}
\hline Preguntas relacionadas con el conocimiento & Frecuencia & $\%$ & $\mathrm{IC}=95 \%$ \\
\hline \multicolumn{4}{|l|}{ P14: Conocimiento de traumas según su severidad } \\
\hline Conoce & 7 & 15 & $4-26$ \\
\hline No conoce & 38 & 84 & $73-95$ \\
\hline \multicolumn{4}{|l|}{ P17 : Área de conocimiento } \\
\hline Endodoncia & 24 & 63 & $38-68$ \\
\hline Cirugía & 20 & 44 & $29-59$ \\
\hline Periodoncia & 1 & 2 & $2-6$ \\
\hline
\end{tabular}

Fuente: elaboración propia

Tabla 3. Descriptivos de actitudes y experiencia en caso de trauma dental en estudiantes de la Escuela de Odontología, Universidad del Sinú, Cartagena, 2015

\begin{tabular}{|c|c|c|c|}
\hline Preguntas relacionadas con las actitudes & Frecuencia & $\%$ & $\mathrm{IC}=95 \%$ \\
\hline \multicolumn{4}{|l|}{ P1: Actitud en subluxación } \\
\hline Favorable & 29 & 64 & $49-78$ \\
\hline No favorable & 16 & 35 & $21-21$ \\
\hline \multicolumn{4}{|l|}{ P3: Actitud en fractura radicular } \\
\hline Favorable & 16 & 35 & $21-50$ \\
\hline No favorable & 29 & 64 & $49-78$ \\
\hline \multicolumn{4}{|l|}{ P4: Actitud en avulsión } \\
\hline Favorable & 15 & 33 & $19-47$ \\
\hline No favorable & 30 & 66 & $52-80$ \\
\hline \multicolumn{4}{|l|}{ P8: Actitud en fractura coronal no complicada } \\
\hline Favorable & 13 & 28 & $15-42$ \\
\hline No Favorable & 32 & 71 & $57-84$ \\
\hline \multicolumn{4}{|l|}{ P9: Actitud en fractura coronal no complicada ápice abierto } \\
\hline Favorable & 21 & 46 & $31-61$ \\
\hline No Favorable & 24 & 53 & $38-68$ \\
\hline \multicolumn{4}{|l|}{ P15: Pasos a seguir en una infracción } \\
\hline Favorable & 10 & 22 & $9-34$ \\
\hline No favorable & 35 & 77 & $65-90$ \\
\hline \multicolumn{4}{|l|}{ P16: Experiencia en clínica } \\
\hline Con experiencia & 2 & 4 & $1-10$ \\
\hline Sin experiencia & 43 & 95 & $89-10$ \\
\hline
\end{tabular}

Fuente: elaboración propia

El nivel conocimiento de trauma dental fue considerado aceptable en el $64,4 \%$ de los sujetos. En cuanto al nivel de actitudes, fue desfavorable en el $82 \%$. Se evidenció que no existe una asociación estadísticamente significativa entre las variables sociodemográficas y el nivel de conocimientos y actitudes referentes a trauma dental (todos los valores $\mathrm{p}>0,05$; Tabla 4 ). 
Tabla 4. Distribución para nivel de conocimientos, actitudes de trauma dental y su asociación con variables sociodemográficas

\begin{tabular}{|c|c|c|c|c|c|}
\hline \multirow{2}{*}{$\begin{array}{c}\text { Características } \\
\text { sociodemográficas }\end{array}$} & \multicolumn{3}{|c|}{ Nivel de conocimientos } & \multirow{2}{*}{$\begin{array}{c}\text { Chi } \\
\text { cuadrado }\end{array}$} & \multirow{2}{*}{$\mathbf{P}$} \\
\hline & Escaso Frec. (\%) & Aceptable Frec. (\%) & Total Frec. (\%) & & \\
\hline \multicolumn{6}{|l|}{ Edad } \\
\hline $21-23$ & $8(17,77)$ & $20(44,44)$ & $28(62,22)$ & \multirow{3}{*}{1,8} & \multirow{3}{*}{0,3} \\
\hline $24-26$ & $7(15,55)$ & $7(15,55)$ & $14(31,11)$ & & \\
\hline$\geq 27$ & $1(2,22)$ & $2(4,44)$ & $3(6,66)$ & & \\
\hline \multicolumn{6}{|l|}{ Sexo } \\
\hline Femenino & $10(22,22)$ & $19(42,22)$ & $29(64,44)$ & \multirow{3}{*}{0,04} & \multirow{3}{*}{0,8} \\
\hline Masculino & $6(13,33)$ & $10(22,22)$ & $16(35,55)$ & & \\
\hline \multirow[t]{3}{*}{ Total } & $16(35,5)$ & $29(64,4)$ & $45(100)$ & & \\
\hline & \multicolumn{3}{|c|}{ Actitudes } & & \\
\hline & Desfavorable & Favorable & Total & & \\
\hline \multicolumn{6}{|l|}{ Edad } \\
\hline $21-23$ & $23(51,11)$ & $5(11,11)$ & $28(62,22)$ & \multirow{3}{*}{0,6} & \multirow{3}{*}{0,7} \\
\hline $24-26$ & $12(26,66)$ & $2(4,44)$ & $14(31,11)$ & & \\
\hline$\geq 27$ & $2(4,44)$ & $1(2,22)$ & $3(6,66)$ & & \\
\hline \multicolumn{6}{|l|}{ Sexo } \\
\hline Femenino & $23(51,11)$ & $6(13,33)$ & $29(64,44)$ & \multirow{3}{*}{0,4} & \multirow{3}{*}{0,4} \\
\hline Masculino & $14(31,11)$ & $2(4,44)$ & $16(35,55)$ & & \\
\hline Total & $37(82,2)$ & $8(17,7)$ & $45(100)$ & & \\
\hline
\end{tabular}

Nota. No existió ninguna asociación estadísticamente significativa.

Fuente: elaboración propia

\section{Discusión}

El presente estudio permitió acceder a una aproximación del nivel de conocimientos, actitudes y experiencia en traumatismos dentales que tienen los estudiantes de último año en una escuela odontológica privada.

La preparación académica en el pregrado de Odontología requiere de la adquisición de conocimientos, y este nivel de aprendizaje o de eficacia en la consecución de los objetivos curriculares depende del progreso de los estudiantes en el proceso académico al cursar las diversas asignaturas y realizar ejercicios en la práctica clínica, bajo la supervisión de los profesores [17]. Además, es importante destacar la necesidad de asumir habilidades para adaptarse a los desafíos de la práctica cotidiana, los cuales varían en los países de acuerdo con las realidades sociales y económicas [18]. Por esta razón, se considera que los conocimientos y las actitudes están de alguna manera relacionados tanto con el entorno cultural, como con los programas académicos y las políticas institucionales.

Entonces, dado que el presente estudio permitió recolectar información de estudiantes de una institución privada, se recomienda en estudios futuros contrastar entre instituciones públicas y privadas los niveles de conocimiento y actitudes en los estudiantes, y la posibilidad de extender la investigación de manera multicéntrica hacia otras ciudades.

La formación en conceptos y teoría en la temática de traumatismo dental alcanza tanto a profesionales de la salud, como a padres de familia, profesores y cuidadores para el caso de niños en edad escolar, generando múltiples estudios para identificar conocimientos, actitudes y prácticas en torno a estas situaciones clínicas, con énfasis en la atención odontológica de traumatismos dentales tipo avulsión [19, 20]. Esto quizá porque el traumatismo facial (representado por dientes fracturados, desplazados o perdidos) puede tener importantes 
efectos negativos funcionales, estéticos y psicológi$\cos [21]$ en las personas. Sobre todo, es posible que la avulsión (desarticulación del diente con un desplazamiento completo fuera del alvéolo) [22] sea el trauma que genera un mayor impacto.

Por otro lado, es posible que los estudios se enfoquen mucho más en odontólogos graduados, porque la situación clínica requiere atención odontológica inmediata al implicar un trauma que genera sangrado tras la ruptura del paquete vasculonervioso [20], lo cual lo convierte en una situación estresante para padres y/o cuidadores, quienes al no saber cómo proceder, trasladan a los niños a la atención hospitalaria o profesional.

Sin embargo, en la presente investigación, se procedió a la valoración del conocimiento y las actitudes en los estudiantes respecto al protocolo para este tipo de traumatismo, considerando la posibilidad de que los estudiantes puedan ser consultados respecto a qué hacer en una de estas situaciones, debido a que realizan sus prácticas es una clínica odontológica que es parte de la misma institución de educación superior en la que estudian.

En referencia a conocimientos específicos para trauma dental, la mitad de los estudiantes no conocen los diferentes tipos de luxación, lo que sería importante cambiar si se toma en cuenta que es un traumatismo dental muy frecuente según los resultados de Rhouma et al. [23] y Vuletic et al. [24] - No obstante, en términos generales, el nivel de conocimiento reportado se consideró aceptable en más de la mitad de los sujetos valorados. Estos resultados son similares a los reportados por Al-Shamiri et al. [25] en el 2015 (Arabia Saudita), por Limbu et al. [26] en el 2014 (Nepal), y por Yanxiang y Yi [27] en el 2010 (China). Algunos estudios discrepan de estos resultados y evidencian un mayor nivel de conocimientos; es el caso de los reportes de Abu-Dawoud et al. [12] en el 2007 (Kuwait) y de Granville [28] en el 2009 (Brasil); o presentan muy bajos niveles de conocimientos, como en los hallazgos de Kostopoulou et al. [13] en el 2005 (Inglaterra), de Pedrini et al. [29] en el 2011 (Brasil) y de Upadhyay et al. [30] en el 2012 (Nepal).

Se destaca que existe una diversidad en los resultados para nivel de conocimientos respecto a traumatismo dental, aunque es realmente preocupante que la mayoría de estos estudios mencionados han sido desarrollados por profesionales ya graduados, una evidencia de que posiblemente el problema supera el escenario académico y que podría ser común en el ejercicio de la práctica odontológica.

Debido a lo anterior, sería recomendable el abordaje de las limitaciones en el conocimiento del traumatismo dental desde el escenario educativo para luego traducirlo en su aplicación dentro del escenario laboral de los futuros odontólogos. En torno a esto, se podrían tomar en consideración aspectos como los contenidos curriculares de los planes de estudio, pues existe la posibilidad de que en esta investigación los resultados positivos en el nivel de conocimiento obedezcan a que los estudiantes de último año han cursado un plan académico formado por una serie de asignaturas teóricas desde cuarto hasta octavo semestre de las áreas disciplinares de periodoncia, cirugía y endodoncia.

En concreto, más de la mitad de los estudiantes manifestaron que los conocimientos adquiridos sobre trauma dental provienen de las asignaturas del área de endodoncia, y es que en octavo semestre los estudiantes cursaron la asignatura de traumatología, lo que constituye una reciente experiencia en la recepción de conceptos y teoría que podría influir en la retentiva de estos conocimientos.

En referencia a las actitudes, hubo resultados generales cuestionables para la atención de trauma dental, y se destacan las actitudes desfavorables que asumen los estudiantes en caso de fractura de corona no complicada sin ápice abierto e infracción, lo que resulta una situación importante pues se ha considerado que estos tipos de traumatismos son muy frecuentes, según los estudios de Sari et al. [31] y Pérezy Aramís [32] en el 2014, y los de Martins Roleira Marinho et al. [33] y Yero Mier et al. [34] en el 2013, de modo que los estudiantes deberían tener actitudes favorables para afrontar la atención de tales casos.

Para la particularidad del trauma tipo avulsión, más de la mitad de los estudiantes no asumirían la mejor actitud en caso de tener que atenderlo, lo cual coincide con lo expuesto por Fujita et al. [35] en el 2014 (Japón) a partir de estudiantes de Odontología. Otros autores como Abdullah et al. [36], en el 2016 (Malasia), reportan cómo, pese a que $46 \%$ de los odontólogos generales valorados obtuvo puntajes favorables en la atención de dientes avulsionados, no son resultados totalmente satisfactorios, por lo que sugirieron la mejora tanto del conocimiento como del ejercicio de la práctica clínica. Así mismo, los autores del presente estudio recomiendan que es necesario utilizar algunas 
estrategias educativas complementarias que permitan capacitar a los estudiantes para mejorar las actitudes ante la atención de estas situaciones clínicas.

La existencia de actitudes desfavorables en la presente investigación quizá se puede relacionar con la poca experiencia referida en la atención de traumatismo dentoalveolar, pues menos del 5\% de los estudiantes ha tenido que asumir la atención de esta situación clínica, lo cual a su vez podría estar relacionado con una poca casuística. De tal modo, es posible tomar en cuenta que a menor número de casos que se presenten en el escenario clínico, menor va a ser la posibilidad de ganar experiencia. Entonces, sería conveniente revisar, en el plan académico y la planeación interna, los escenarios de práctica y de rotaciones clínicas disponibles, a fin de propiciar un mayor contacto de los estudiantes con la atención de estos casos; incluso se propone hacer rotaciones clínicas en espacios de atención de urgencias o habilitando una unidad destinada para estos fines en la Escuela de Odontología.

Pese a lo anterior, algunos autores describen que la experiencia, las actitudes y los conocimientos sobre traumatismo dental son independientes. Tal es el caso de Ulusoy et al. [37], en el 2012 (Turquía), pues luego de valorar profesionales en diferentes hospitales del servicio de urgencias, aun con una experiencia en atención de trauma dental en más de la mitad de los casos, se encontró con un nivel de conocimientos insuficiente en cerca del $50 \%$ de los profesionales.

En lo concerniente al efecto de las características demográficas en los conocimientos y las actitudes, Sen et al. [38], en el 2014, en su estudio referente a conocimientos de atención de trauma dental en maestros, identificaron que no hubo diferencias estadísticamente significativas entre el nivel de conocimientos, la edad y el sexo. En el caso particular de los estudiantes de Odontología, la influencia de ser hombre o mujer en el nivel de conocimientos resulta ser interesante, pues incluso la formación académica adicional sobre atención del trauma dental varía entre los dos sexos y favorece a los hombres, tal como lo reporta Al-Shamiri et al. [25], en el 2015 (Arabia Saudita), quien a su vez obtuvo mejores resultados de las mujeres en cuanto a las actitudes.

Pese a lo anterior, en este estudio, las características de edad y sexo no influyeron en los resultados para conocimientos y actitudes, considerando una edad distribuida en rangos que no fueron muy distantes entre sí y que más de la mitad de los estudiantes eran mujeres.

Es importante reconocer aquí algunas limitaciones como la posible existencia del sesgo de selección, pues quienes se vincularon al estudio a lo mejor tendrían mayor interés en la temática de trauma dental; además, el diseño transversal solo permite asumir los resultados para un único momento en el tiempo, por lo cual sería interesante desarrollar una investigación en la que se pudiera dar seguimiento a los estudiantes hasta que culminen sus estudios e incursionen en el ámbito profesional, a fin de identificar: ¿cuáles son los cambios y los progresos en conocimientos y actitudes respecto a traumatismos dentales?; ¿cuáles son los semestres en los que se evidencia mejor comprensión de conceptos, mejor nivel de conocimientos y actitudes más favorables?, y ¿cuál es el efecto que tiene la experiencia clínica con el paso de los años en los conocimientos y las actitudes de los sujetos?

\section{Conclusiones}

Los sujetos de estudio poseen conocimiento aceptable respecto a trauma dental y actitudes poco favorables para asumir la atención. Quizá, esto se relaciona con la poca experiencia clínica evidenciada en los estudiantes, lo que genera la necesidad de realizar las intervenciones educativas necesarias desde el área de la teoría hasta el escenario de la práctica, promoviendo rotaciones en escenarios clínicos donde se brinde la oportunidad de que los estudiantes atiendan este tipo de urgencias con mayor frecuencia, a fin de posibilitar la aplicación práctica de los conocimientos y el desarrollo de las actitudes para afrontar la atención.

\section{Referencias}

[1] Martins VM, Sousa RV, Rocha ES, Leite RB, Paiva SM, Granville-García AF. Dental trauma among Brazilian schoolchildren: Prevalence, treatment and associated factors. Eur Arch Paediatr Dent. 2012; 13(5):232-7.

[2] Jamidez Herrera Y, Romero Zaldívar E, Pérez Cedrón R, López Hernández P. Short-term evaluation of traumatized teeth after the application of treatments. AMC [Internet]. 2010 [citado 2017 ene 11]; 14(6):1-10. Disponible en: http://scielo.sld.cu/ scielo.php?script $=$ sci_arttext\&pid=S1025-025520 $10000600010 \& \ln \mathrm{g}=\mathrm{es}$. 
[3] Díaz JA, Bustos L, Brandt AC, Fernández BE. Dental injuries among children and adolescents aged 1-15 years attending to public hospital in Temuco, Chile. Dent Traumatol. 2010;26(3):254-61.

[4] Rodríguez A, Pérez Y, Fernández ME, Vila D, Cuevillas G. Traumas dentoalveolares relacionados con maloclusiones en menores de 15 años. Rev Cubana Estomatol [Internet]. 2011 [citado 2017 ene 11]; 48(3):241-8. Disponible en: http://scielo.sld.cu/scielo.php? script $=$ sci_arttext\&pid=S0034-75072011 000300006\&lng=es.

[5] Norton E, O'Connell AC. Traumatic dental injuries and their association with malocclusion in the primary dentition of Irish children. Dent Traumatol. 2012;28(1):81-6. doi: https://doi.org/10.1111/j. 1600-9657.2011.01032.x.

[6] Azami-Aghdash S, Ebadifard Azar F, Pournaghi Azar F, Rezapour A, Moradi-Joo M, Moosavi A, et al. Prevalence, etiology, and types of dental trauma in children and adolescents: Systematic review and meta-analysis. Med J Islam Repub Iran. 2015;29(4):234.

[7] Krishna A, Malur MH, Swapna DV, Benjamin S, Deepak CA. Traumatic dental injury - An enigma for adolescents: A series of case reports. Case Rep Dent. 2012;2012:756526. doi: https://doi.org/10.11 $55 / 2012 / 756526$.

[8] Wennervaldt K, Melchiors J. Risk of perforation using rigid oesophagoscopy in the distal part of oesophagus. Dan Med J. 2012;59(11).

[9] Abdel Jabbar NS, Aldrigui JM, Braga MM, Wanderley MT. Pulp polyp in traumatized primary teeth -A case-control study. Dent Traumatol. 2013;29(5):360-4.

[10] Hegde AM, Kumar KN, Varghese E. Knowledge of dental trauma among mothers in Mangalore. Dent Traumatol. 2010;26(5):417-21.

[11] Al-Obaida M. Knowledge and management of traumatic dental injuries in a group of Saudi primary schools teachers. Dent Traumatol. 2010;26(4): 338-41.

[12] Abu-Dawoud M, Al-Enezi B, Andersson L. Knowledge of emergency management of avulsed teeth among young physicians and dentists. Dent Traumatol. 2007;23(6):348-55.

[13] Kostopoulou MN, Duggal MS. A study into dentists' knowledge of the treatment of traumatic injuries to young permanent incisors. Int J Paediatr Dent. 2005;15(1):10-9.

[14] Divaris K, Barlow PJ, Chendea SA, Cheong WS, Dounis A, Dragan IF, et al. The academic environment: The students' perspective. Eur J Dent Educ. 2008;12(Suppl1):120-30. doi: https://doi.org/10.1111/j.1600-0579.2007.00494.x.
[15] Colombia, Ministerio de Salud. Resolución 8430, Por la cual se establecen las normas científicas, técnicas y administrativas para la investigación en salud (1993 oct 4). Disponible en: http://www.minsalud. gov.co/Normatividad/RESOLUCION\%208430\%20 DE\%20 1993.pdf.

[16] Dental Trauma Guide [Internet]. 2008 [citado 2017 mar 2]. Copenhague: International Association for Dental Traumatology. Disponible en: dentaltraumaguide.org.

[17] García Y, López D, Rivero O. Estudiantes universitarios con bajo rendimiento académico, ¿qué hacer? Rev Edumecentro [Internet]. 2014 [citado 2017 mar 12]; 6(2):272-8. Disponible en: http://scielo.sld.cu/ scielo.php?script $=$ sci_arttext $\&$ pid $=$ S2077-287420 $14000200018 \& \operatorname{lng}=$ es.

[18] Chapper A, Campani SA, Paiva V da S, De Assis C de A, García E, Abreu e Silva FA. Comparison of student satisfaction in public versus private dental schools in Brazil. J Dent Educ. 2007;71(10):1363-9.

[19] Westphalen VPD, Martins WD, Deonizio MDA, Da Silva Neto UX, Da Cunha CB, Fariniuk LF. Knowledge of general practitioners dentists about the emergency management of dental avulsion in Curitiba, Brazil. Dent Traumatol. 2007;23(1):6-8.

[20] Cohenca N, Forrest JL, Rotstein I. Knowledge of oral health professionals of treatment of avulsed teeth. Dent Traumatol. 2006;22(6):296-301.

[21] Dua R, Sharma S. Prevalence, causes, and correlates of traumatic dental injuries among seven-to-twelveyear-old school children in Dera Bassi. Contemporary Clinical Dentistry. 2012;3(1):38-41. doi: https:// doi.org/10.4103/0976-237X.94544.

[22] Jordán F, Sossa H, Estrada JH. Protocolo de manejo de diente permanente avulsionado para el servicio de salud oral de la Fundación Hospital La Misericordia y la Facultad de Odontología de la Universidad Nacional de Colombia, sede Bogotá (2011). Univ Odontol. 2012;31(66):185-210.

[23] Rhouma O, McMahon AD, Welbury R. Traumatic dental injury and social deprivation in five-year-old children in Scotland 1993-2007. BDJ British Dental Journal. 2013;26:214-8.

[24] Vuletic M, Sharicic J, Batinjan G, Trampus Z, Cukovic BI, Hrvoje J. A retrospective study on traumatic dental and soft-tissue injuries in preschool children in Zagreb, Croatia. Bosn J Basic Med Sci. 2014;14(1):12-5.

[25] Al-Shamiri HM, Alaizari NA, Al-Maweri SA, Tarakji B. Knowledge and attitude of dental trauma among dental students in Saudi Arabia. Eur J Dent. 2015;9(4):518-22. doi:https://doi.org/10.4103/13057456.172636 
[26] Limbu S, Dikshit P, Bhagat T, Mehata S. Knowledge of dental interns towards emergency management of avulsed tooth in dental colleges in Nepal. J Nepal Health Res Counc. 2014;12(26):1-7.

[27] Yanxiang Zhao, Yi Gong. Knowledge of emergency management of avulsed teeth: A survey of dentists in Beijing, China. Dent Traumatol. 2010;26(3):281-4.

[28] Granville-Garcia A, Soares JM, De Menezes VA, Bezerra SDA, Torres M, Leite A. Dental avulsion: Experience, attitudes, and perception of dental practitioners of Caruaru, Pernambuco, Brazil. Rev Odonto Ciênc. 2009;24(3):244-8.

[29] Pedrini D, Panzarini SR, Poi WR, Mazza Sundefeld MLM, Ferreira AR. Dentists' level of knowledge of the treatment plans for periodontal ligament injuries after dentoalveolar trauma. Braz Oral Res. 2011;25(4):307-13.

[30] Upadhyay S, Rokaya D, Upadhyaya C. Knowledge of emergency management of avulsed teeth among general dentists in Kathmandu. Kathmandu Univ Med J. 2012;38(2):37-40.

[31] Sari ME, Ozmen B, Koyuturk AE, Tokay U, Kasap P, Guler D. A restrospective evaluation of traumatic dental injury in children who applied to the dental hospital, Turkey. Niger J Clin Pract. 2014;17(5):644-8.

[32] Pérez DM, Aramís MP. Comportamiento de las lesiones traumáticas en niños y adolescentes en un área de salud de Camagüey. Mediciego. 2014;2(20).
[33] Martins Roleira Marinho AC, Manso MC, Colares V, Casimiro de Andrade DJ. Prevalência de traumatismo dentário e fatores asociados em adolescentes no concelho do Porto. Rev Port Estomatol Med Dent Cir Maxilofac. 2013;54(3):143-9.

[34] Yero Mier IM, González Fortes B, Mursulí Sosa M, Cruz Milián MC. Traumatismo dentario 15 a 18 años. Escuela Militar Camilo Cienfuego Sancti Spíritus 2011. Gac Méd Espirit. 2013;15(1).

[35] Fujita Y, Shiono Y, Maki K. Knowledge of emergency management of avulsed tooth among Japanese dental students. BMC Oral Health. 2014;14:34. doi: https://doi.org/10.1186/1472-6831-14-34.

[36] Abdullah D, Soo SY, Kanagasingam S. Knowledge of managing avulsed tooth among general dental practitioners in Malaysia. Singapore Dent J. 2016;37:216. doi: https://doi.org/10.1016/j.sdj.2016.01.001.

[37] Ulusoy A, O* Nder H, Bengi C, Kaya S. Knowledge of medical hospital emergency physicians about the first-aid management of traumatic tooth avulsion. Int J Paediatr Dent. 2012;22(3):211-6.

[38] Sen S, Saha S, Jagannatha GV. Knowledge on management of traumatic dental injuries among schoolteachers in Lucknow. J Indian Assoc Public Health Dent. 2014;12(4):312-6. doi: https://doi.org/10.410 3/2319-5932.147676. 